\title{
Symmetry Transformations and Information Growth in Biological Morphogenesis and Evolution
}

\author{
Abir U. Igamberdiev \\ Department of Biology, Memorial University of Newfoundland, St. John’s, NL A1B3X9, Canada; E- \\ mail: igamberdiev@mun.ca, Tel.: +1-709-864-4567; Fax: +1-709-864-3018
}

Accepted:

\section{Introduction}

The processes of ontogenesis and evolution are based on perpetual changes in interpretation of information in the changing world. These changes lead to rescaling of internal time and of the spatial structure. Relative independence of the biological form from underlying molecular processes (including gene expression) can be considered as one of the major biological principles. It allows studying morphology as the transformation of geometry. This approach philosophically arises to Plato. D’Arcy Wentworth Thompson (1917) in his famous book “On Growth and Form” showed that transformations of coordinate system correspond to generation of forms specific for concrete biological species. He also mentioned that "morphology is not only a study of material things and of the forms of material things, but has its dynamical aspect, under which we deal with the interpretation, in terms of force, of the operations of Energy".

The basic understanding of the space and symmetry of biological forms comes from the observation that it is essentially curvilinear, being more diverse and complex than any physical space-time. Leo Berg (1922) in his theory of nomogenesis claimed that the laws of evolution are based on the objective rules of symmetrical transformations of form that are independent from adaptability and natural selection. This approach is essentially Platonic. The concept that form is not "encoded" but rather unfolded within the whole organization can be discussed in relation to the basic principle that the whole system cannot be reduced to its internal description (genetic structures). To understand the actualization of form from the underlying phenomena, we need to go beyond the concept of genetic determination and analyze fundamental principles of biological organization based on percolation between different levels of physical reality from the basic quantum phenomena to the upper levels (Conrad, 1996). This corresponds to the actualization of potentia and represents the Aristotelian approach to understanding the biological form. Genes are, in fact, the subsets of metabolites in the 
autopoietic structure of biological system (Igamberdiev, 1999) stably reflecting proteins and their functions, while form appears beyond this reflection. It is a projection into 3D of the whole structure consisting of metabolites, enzymes and genes that bootstrap each other.

\section{Nomothetic Constraints in the Generation of New Structures during Ontogenesis}

There is no mathematical preference to any curvature. The existence of metrics, according to Riemann (1868), can be explained by certain external physical forces of connection that are applied to it. This is an example of imposing limitations on mathematical forms through application of physics, which is essentially the science about the limits of computation appearing to us as physical laws (Igamberdiev, 2007). These forces of connection are related to the discreteness of space, and form its actual observed structure. When there is no "active" observer concerned, the modeling of space-time results in the Euclidean geometry of the Newtonian space with the single unitary Cartesian coordinate system. When we consider the observer "actively" measuring space-time by signals with finite velocity, we come to Einstein's relational space-time but with the equivalence of all coordinate systems. And when the effect of measurement is concerned, we come to the choice (generation) of a coordinate system and its transformation according to the search of a stable (homeostatic) state. This generates various structures described by the non-Euclidean geometry.

The development of morphogenetic patterns can be viewed as a sequence of unfolding patterns originating from previous states. We can study the unfolding pattern without reference to underlying phenomena. Nevertheless there should be something in the pattern of unfolding that refers to the system's internal activity. We can distinguish an internally developing generic process from an external non-generic phenomenon through certain proportions in the generated structure, which represent the limits of iteration exhibiting an internal process. Any internal choice exhibits a structure of the mixing the notion of indicating an element with the act of indicating a set consisting of elements (Igamberdiev, 2004). Rosen (1993) noted that physically embodied reflective loops have certain objective parameters that include the values that can be redundantly repeated. This leads to a topological inevitability of certain developmental processes so that the topological singularities inevitably emerge in biological morphogenesis, and are retained and transformed during pattern formation (Isaeva et al., 2014).

Certain transition rules are used recursively along time and generate fixed points (the points of coincidence of the image and its reflection). During this process, the development is collapsed into the fractal spatiotemporal structure (Gunji et al., 1997). According to this approach, the fixed point $x$ for the operation of determination of $\mathrm{A}_{\text {and }} \mathrm{A}^{-}$, denoted by $\mathrm{F}$ can be expressed as an infinite recursion, $x=$ $F(F(F(\ldots F(x) \ldots))$ ), by mapping $x=F(x)$ onto $x=F(x)$ and presented as a point in two-dimensional space. The operation of $F$ is the contraction in a two-dimensional domain, indicating either $\mathrm{A}$ or $\mathrm{A}^{-}$ (Kitabayashi et al., 1999). If validity of $\mathrm{A}$ is denoted by $m$, the invariance of validity with respect to contraction is expressed as $f(m) * m=$ constant, where $m$ is the value of validity and $f(m)$ is the probability of $m$. If distribution of $f(m)$ does not have an off-set peak, $m$ directly means the rank. Then $f(m) * m=c$ represents what is called the Zipf's law, i.e. $\log (f(m))=-\log (m)+c$.

In biological morphogenesis, the preceding motif unit is transferred into the subsequent one by a certain fixed similarity transformation g, i.e., $F_{n+1}=g * F_{n}$. The finite representation of actualization forms a coordinate scale inherent to any individual morphological form. These coordinate scales can 
be transformed by simple recursive rules via rescaling (Thompson d'Arcy, 1917). The limit of actualization fits optimality of the structure being actualized, thus it provides the existence of optimal solutions for design. If we take the simplest and most general way of transformation when a new domain is composed of two previous (two-dimensional transformation), the two sequential values are composed to get the third value. Thus the next value is composed from the two previous values when they are memorized: $F_{n+2}=F_{n}+F_{n+1}$. This means that certain recursive limits appear as fundamental parameters of memorization in the course of unfolding process. In many cases of biological morphogenesis the configuration of golden section is realized as the limit $(n \rightarrow \infty)$ of this process:

$$
\Phi=\lim \left(F_{n+1} / F_{n}\right)=(1+51 / 2) / 2=1.618 \ldots
$$

The solution for $F_{n}$ depends only on the number of recursions addressing the fixed point and described by the Fibonacci number formula:

$$
F_{n}=\left[\left(1+5 \frac{1}{2}\right)^{\mathrm{n}}-\left(1-5 \frac{1}{2}\right)^{\mathrm{n}}\right] /\left(2^{\mathrm{n}} \times 5^{1 / 2}\right)
$$

The Fibonacci numbers represent possible solutions for morphogenetic problems, as numbers of ways of picking sets in recursive process with the formation of corresponding spatial patterns. Important series (referring to three-dimensional unfolding) appear when three neighboring elements $F_{n}, F_{n+1}, F_{n+2}$ of the Fibonacci are taken as lengths of three sequential segments (as appeared in the sequential past $(t-1)$, present $(t)$ and future $(t+1)$ times). They represent the ratio defined as the wurf having its "golden wurf” limit W (Petukhov, 1989):

$$
W=\lim \left(F_{n}+F_{n+1}\right)\left(F_{n+1}+F_{n+2}\right) /\left[F_{n+1}\left(F_{n}+F_{n+1}+F_{n+2}\right)\right]=\Phi^{2} / 2=\left(3+5 \frac{1}{2}\right) / 4=1.309
$$

The value of golden wurf arises from the combination of three sequential segments with the values 1, $\Phi$ and $\Phi^{2}$, i.e. it follows from the memorization of limits of recursion in the Fibonacci series (Petukhov, 1989). The golden section and the golden wurf constants represent fundamental values of infinite recursion, when the next element is formed by the operation on the sequentially appearing elements within the reflective loop, and occur in many morphogenetic patterns.

\section{Nomothetic Constraints in the Generation of New Structures during Evolution}

Nomothetic constraints of the evolutionary process and the evolutionary variability follow certain rules that can be called nomothetical laws and analyzed as symmetrical transformations (Meyen, 1973). E.g., in leaf evolution some transitions are possible, other transitions are less probable and some are prohibited. The probability of transition depends on stage of development, on evolutionary position of species and on their geographical location. The sequence of evolutionary transitions of a certain structure can be viewed as a recursion process called "refrain” by Meyen (1973) and lead to generation of repeated polymorphic sets.

This type of recursion follows the principle of self-similarity in which the quantitative invariants may not conserve but the qualitative (e.g. topological) characteristics will be preserved. The simplest and most general way of transformation occurs when the new domain is composed of two previous 
(Igamberdiev, 2004). In this process, the evolving state is determined by the two contradictory values of the system separated by time interval, and the value in time future is acquired after addressing them. Two contradictory statements taken as sequential values separated by time interval are composed to get the third statement. Thus the next statement (quantitatively modeled as having correspondent value) is composed from the two previous statements when they are memorized as real numbers in the course of recursive process: $F_{n+2}=F_{n}+F_{n+1}$. In this case, certain recursive limits will appear as fundamental canons of perfection formed as memorization within reflective loops, corresponding to symmetry transformations. The notion of golden section $\left(\Phi=\lim F_{n+1} / F_{n}\right)$, which commonly appears in biological patterns formation, follows from this type of memorization (Igamberdiev, 2004).

Symmetry transformations in morphogenesis and evolution are determined by the pre-existing information that shapes possible combinatorial limits. In the course of biological transformations, the changes are introduced in genomes, but still the changes in interpretation remain more meaningful than the changes in genetic information itself. The process of interpretation of information includes a complementary interaction of the digital information (encoding) with the non-digital information (templating) which reads (decodes) the code (i.e., realizes the reflective action). The interaction between these two types of information non-recursively forms an interpretant for the semiotic system of living being. The information based on specific recognitions triggering the dynamical energy-driven processes appears as non-digital; the transfer of digital information is realized within hypercycles and corresponds to operation of the genetic code. Changing of interpretation corresponds to symmetry transformation in biological system.

\section{Conclusion}

The systems having embedded internal description are, according to Rosen, anticipatory in the sense that the embedded description generates models of their behavior. If the model does not provide a correct result, they can evolve due to acquisition of new statements inside the embedded description that overcome limitations of the existing model. Generation of new statements has its logical precondition in the incompleteness of internal formal system of biosystem. The newly generated statements are empowered by meanings in the changing environment. The growth of complexity generates nomothetic patterns of spatiotemporal structures of evolving systems. These patterns reproduce certain optimal proportions such as the golden ratio, during their realizations. Thus living systems have their internal times, and in the course of morphogenesis and evolution, time rescaling takes place. The processes of time rescaling are triggered in epigenetic evolution by the biospheric parameters causing time shift in metabolic processes and in genome mobility (Igamberdiev, 2014). This corresponds to symmetry transformations in which the changes of interpretation of information occur that drive further changes towards the evolutionary growth of complexity.

\section{References}

1. Berg, L.S. Nomogenesis; or, Evolution Determined by Law. M.I.T. Press, Cambridge, 1922-1969.

2. Conrad, M. Cross-scale information processing in evolution, development and intelligence. Biosystems 1996, 38, 97-109. 
3. Gunji, Y.-P., Ito, K., Kusunoki, Y. Formal model of internal measurement: Alternate changing between recursive definition and domain equation. Physica D 1997, 110, 289-312.

4. Igamberdiev, A.U. Quantum computation, non-demolition measurements, and reflective control in living systems. Biosystems 2004, 77, 47-56.

5. Igamberdiev, A.U. Physical limits of computation and emergence of life. Biosystems 2007, 90, 340-349.

6. Igamberdiev, A.U. Time rescaling and pattern formation in biological evolution. Biosystems 2014, 123, 19-26.

7. Isaeva, V.V., Kasyanov, N.V., Presnov, E.V. Topological and physical constraints and directions in biological morphogenesis. Biosystems 2012, 109, 280-298.

8. Kitabayashi, N., Kusunoki, Y., Gunji, Y.P. The emergence of the concept of a tool in foodretrieving behavior of the ants Formica japonica Motschulsky. Biosystems 1999, 50, 143-156.

9. Meyen, S.V. Plant morphology in its nomothetical aspects. Botanical Review 1973, 39, $205-260$.

10. Petukhov, S.V. Non-Euclidean geometries and algorithms of living bodies. Computers and Mathematics with Applications 1989, 17, 505-534.

11. Riemann, B. Collected Papers. Kendrick Press, Heber City, UT, 1868-2004.

12. Rosen, R. Drawing the boundary between subject and object - comments on the mind-brain problem. Theoretical Medicine 1993, 14, 89-100.

13. Thompson D’Arcy, W. On Growth and Form. Cambridge University Press, London, 1917.

(C) 2015 by the author; licensee MDPI and ISIS. This abstract is distributed under the terms and conditions of the Creative Commons Attribution license. 